\title{
LAS INNOVACIONES DIGITALES EN EDUCACIÓN Y LA IRRUPCIÓN DE UNA PEDAGOGÍA INFORMÁTICA
} Digital innovations in education and the emergence of computer pedagogy

\author{
Claudio Rama ${ }^{1}$ \\ Universidad de la Empresa, Uruguay
}

\section{RESUMEN}

El presente artículo analiza los cambios en los procesos de enseñanza derivados de las innovaciones producidas gracias a la digitalización y plantea la irrupción de una pedagogía de estos contextos tecnológicos. Primero, reconoce los procesos de innovación en la lógica de la dinámica social; luego analiza aquellos que acontecen en la educación; por último, se centra en el aumento de la intensidad de los recursos de aprendizaje y el uso de aplicaciones informáticas como la base que soporta una nueva pedagogía. El trabajo sostiene que la dinámica innovativa digital, al centrarse en recursos de aprendizaje, cambia las lógicas del trabajo educativo y expande la sustitución del trabajo vivo (trabajo docente directo) por el trabajo muerto (trabajo docente previo) como eje de los aprendizajes. En esta realidad en curso, formula que la digitalización contribuye a una mayor convergencia entre las modalidades -y probablemente borrar las viejas fronteras de distancia y presencialidad en la educación - y que gesta la posibilidad de una nueva dinámica

Palabras Clave: Innovación, digitalización, aprendizaje, recursos de aprendizaje, pedagogía informática

\section{Abstract}

This article analyzes the changes in the teaching processes derived from the innovations produced through digitization and suggests the emergence of pedagogy of these technological contexts. Upon recognition of the innovation processes in the logic of social dynamics; it analyzes its processes in education, focused on the increasing of intensity of learning resources and the use of computer applications that are considered as the base that supports a new pedagogy. The paper argues that the innovative digital dynamics being focused on learning resources, changes the logics of educational work and expands the replacement of living labor (direct teaching work) by dead labor (previous teaching job) as the core of learning. Immersed in this ongoing reality, it states that digitization contributes to a greater convergence between modalities and it will probably remove the old distance borders and the need of face-to-face education. It also implies the possibility of a new teaching and learning dynamics in digital environments defined as computer pedagogy.

Keywords: Innovation, digitization, learning, learning resources, computer pedagogy.

1 Investigador y consultor en educación superior. Economista (UCV); Especialista en Marketing (UCUDAL); Especialista en Educación a Distancia (UNA); Master en Gerencia Educativa (UJMV); Doctor en Educación (UNESR); Doctor en Derecho (UBA) y tres postdoctorados (UNICAMP, UFF, UNESR). Actualmente está terminando otro postdoctorado en la Universidad de Buenos Aires. Fue Director del Instituto Internacional de la UNESCO para la Educación Superior en América Latina y Caribe (IESALC). En Uruguay, fue Director del Instituto Nacional del Libro, Director del Sistema Nacional de Televisión y Vicepresidente del Servicio de Difusión Radio, Televisión y Espectáculos (SODRE). Actualmente es Decano de la Facultad de Ciencias Empresariales de la Universidad de la Empresa (UDE), investigador categorizado en el Sistema Nacional de Investigadores, Director del Observatorio de la Educación Virtual en América Latina y El Caribe (Virtual Educa - OEA), y profesor invitado en varios programas de postrado (UDE, UA, UNISUL, UNLZ). claudiorama@gmail.com 


\section{INTRODUCCIÓN}

La virtualización de la educación constituye la mayor innovación actual de los procesos educativos $^{2}$. La virtualización es una de las tendencias más fuertes de los procesos educativos a escala global (Rama, 2007, 2010). Esta remite a cambios tecnológicos en los modelos societarios, con nuevas formas de gestionar y procesar la información y nuevas concepciones del aprendizaje centradas en la construcción de competencias. Los factores tecnológicos se han constituido en los componentes más dinámicos en la educación a distancia tradicional y de las transformaciones de los sistemas universitarios, con nuevos paradigmas educativos, institucionales, actores, formas de gestión y lógicas económicas. Entre esos cambios se concibe el nacimiento de una pedagogía informática, cuyo análisis es el centro de este ensayo.

Desde los estudios sobre las máquinas de enseñar y la instrucción programada (Skinner, 1979) o desde el uso de medios de comunicación en el aprendizaje, los especialistas reconocen el alto rol de las tecnologías en todas las modalidades educativas. Hay una larga discusión alrededor de los enfoques deterministas en materia tecnológica con distintas visiones sobre el grado de incidencia de los componentes tecnológicos en los procesos educativos (Tedesco, 2008). Sin embargo, los debates actuales y la investigación asociada a la eficacia instruccional de las nuevas herramientas y equipamientos digitales tienden a mostrar las crecientes correlaciones entre industrias culturales y las tecnologías de información y comunicación (TIC) y los resultados de los aprendizajes en las modalidades a distancia, e incluso las propias configuraciones organizacionales de las instituciones

2 La sola revisión de las revistas dedicadas a la educación a distancia, confirma esta realidad a través de trabajos y análisis descriptivos de su incorporación $\mathrm{Al}$ respecto se pueden ver los diversos trabajos de la Revista Iberoamericana de Educación a Distancia (RIED) (AIESAD) (http://ried.utpl.edu.ec/es/node/464), de la Revista Argumentos de la Universidad Virtual de Guadalajara (http://www.udgvirtual.udg.mx/ apertura/index.php/apertura3/index), de la Revista Mexicana del Bachillerato en Línea (http://www.journals.unam.mx/index.php/rmbd), de la Revista Educación Siglo XX1 de la Universidad Nacional de Educación a Distancia (UNED) (http://www.rerce.es/). Para ver el impacto estadístico y educativo en un caso, Brasil, ver Censo EAD. Brasil (2010), ABED, Pearson, Secretaria de Educaco a Distancia. Para ver el impacto nacional Litto, Fredric y Formiga, Marcos (2009). Educacao a distancia o estado da arte. ABED, Pearson: SP. Para ver impactos en diversos países y temas, ver los 11 informes desde el 2008 del Observatorio de la Educación Virtual de América Latina y el Caribe de Virtual Educa (http://virtualeduca.org/observatorio.htm) educativas (Youssef \& Ramirez, 2011).

En el actual contexto de la revolución digital, los viejos intentos de construir una dinámica de enseñanza a través de máquinas, como se intentó en la década del 50, han sido desestimados y la programación informática se ha constituido en el instrumento para promover esos fines. En los años 50 se desarrollaron y se pusieron a la venta múltiples modelos de máquinas de enseñar que quedaron rápidamente obsoletas por su simpleza, la complejidad de sus objetivos para las rudimentarias tecnologías y los altos costos de realizar la programación del funcionamiento de las propias máquinas (del hardware) para estructurar los aprendizajes (Skiner, 1979). Sin embargo, con la irrupción de la programación informática y los recursos de aprendizajes, y por ende del desarrollo de máquinas herramientas accionadas por la programación informática (Coriat, 1992), dichas reflexiones comenzaron a ser replanteadas.

La virtualización ha transformado las modalidades tradicionales de la educación a distancia, como se ha analizado por algunos autores (Bates, 2001; Daniel, 2007; Aretio, 2007; Tiffin \& Rajasingham, 2007). A la par, se están transformando las aulas y la dinámica de enseñanza y aprendizaje presencial, por ejemplo gracias al uso de plataformas interactivas virtuales o ambientes virtuales de aprendizajes, más conocidas por sus siglas del inglés como LMS (Learning Management System), o de otras aplicaciones informáticas de aprendizaje mediante la realidad virtual simulada de la práctica profesional. También se destaca el uso de ambientes virtuales a través de una evaluación automatizada de los aprendizajes y sobre todo mediante la digitalización de los recursos de aprendizaje y el acceso en red a través de internet. Este es un proceso de innovaciones que modifica la educación presencial y que sienta las bases de una convergencia de las modalidades tradicionales de enseñanza presencial y a distancia. Esto impulsará múltiples reformas y reingenierías en las que aumenta el componente de gasto tecnológico en las instituciones y perfilan un enfoque por competencias cambiando el rol de los docentes. Asimismo, modificarán las características de las aulas al abrir un nuevo camino para aumentar la calidad de los aprendizajes asociado al incremento en los 
niveles de digitalización. Esta es la innovación más importante en la educación en lo que se ha considerado como "la reforma de la virtualización y el nacimiento de la educación digital" que atraviesan todos los sistemas educativos (Rama, 2012).

\section{LA INNOVACIÓN COMO BASE DEL CAMBIO SOCIAL}

La innovación es el eje central de la dinámica económica, social, y crecientemente, de la educación. Shumpeter conceptualizó una dinámica de la economía al centrar en la innovación tecnológica el principal elemento de la competencia a través de la "creación destructiva", que a la vez que concibe nuevos productos, procesos o servicios, torna obsoletos o deprecia los procesos, capacidades y productos anteriores. Esto está en el centro de la capacidad de crear ganancias extraordinarias al cambiar los niveles competitivos entre las distintas organizaciones (Rosemberg, 2003). Esta lógica de la dinámica económica ha sido la base de la explicación de la expansión del conocimiento, del aumento de la composición orgánica de los procesos productivos y de la expansión del empleo capacitado en las últimas décadas. Correlacionado con ello también ha sido la base de cambios educativos impulsando una mayor articulación entre conocimientos, educación y mercado para construir las capacidades en las personas (Carnoy, 2006). En esta dirección, la innovación comenzó a constituirse también en uno de los ejes de la dinámica educativa, la cual se expresó en múltiples manifestaciones: en nuevas pedagogías, currículos, didácticas y ambientes educativos, así como en el desarrollo de los recursos de aprendizaje. Entre esas innovaciones sobresale crecientemente la incorporación de componentes tecnológicos fundamentalmente digitales para apoyar y guiar la enseñanza y el aprendizaje (Montoya, 2004).

Los enfoques primigenios de Shumpeter tuvieron un sesgo hacia un relativo determinismo tecnológico como base de la innovación y la competencia capitalista, pero enfoques más contemporáneos formulados dentro de esta escuela de pensamiento, han tomado en consideración las estructuras sociales, las culturas, las infraestructuras, los procesos de creación de conocimientos y el capital humano o social, como componentes básicos y determinantes de la innovación (Pérez 1996, 2004). Esta autora refiere que una revolución tecnológica es un conjunto de tecnologías, productos e industrias nuevas, capaces de sacudir los cimientos de la economía y de impulsar una oleada de desarrollo a largo plazo, pero cuya introducción impone cambios en toda la estructura social. Asimismo, bajo este enfoque, cambia el rol exclusivo del empresario innovador y se conforman nuevas sintonías entre tecnologías, estructuras y culturas organizacionales, capacidades de gestión y sistemas de enseńanza. Derivado de este paradigma shumpetereano se ha construido el enfoque de la triple hélice (gobierno, empresa y universidad) como centro de la innovación en las últimas décadas. (Albornoz, 2009).

Ello ha ido dirigiendo la innovación desde las áreas tecno-económicas, hacia toda la sociedad y transformando un enfoque con alto determinismo tecnológico de la innovación hacia un multideterminismo integrado y donde la innovación se focaliza en áreas muy diversas (Villa, 2001, Easterly, 2002) ${ }^{3}$. De centrar el acento únicamente en la infraestructura tecnológica se develan múltiples niveles de cambios e innovaciones necesarias y complementarias en toda la estructura de las sociedades, y entre las cuales crecientemente en la educación como ámbito centrado en la formación de recursos humanos y la creación de conocimientos. Muchos de los cambios e innovaciones que se realizan en una amplia línea de ámbitos sociales propender a acompañar y viabilizar la propia transformación en la base tecnológica, en las transformación de los procesos de trabajo o en los determinantes para la creación de la riqueza que requiere más capacidades humanas, nuevas modalidades de movilidad internacionales o políticas más articuladas.

El paradigma shumpetearo ha sido el cimiento para reconceptualizar el rol del conocimiento y este mismo tuvo uno de sus basamentos en el Informe Bush, que en los años 40 analizó cómo la ciencia es la fuente directa de la creación e innovación a través de investigaciones que traen como resultado la generación de nuevas tecnologías.

\footnotetext{
3 En esta ampliación de enfoques se han desarrollado las teorías del capital humano, del capital intelectual y del capital social que acompañan esos cambios conceptuales en los modelos de desarrollo.
} 
(Busch, 1999). Así, la ciencia y la innovación se asociaron más estrechamente, e impulsaron una estructuración particular de la dinámica educativa con la investigación, que articuló nuevas modalidades de articulación entre la academia y las empresas, entre la investigación y el mercado, entre los gobiernos y el financiamiento a la investigación (Aboites y Soria, 2008).

En este marco de innovación shumpetereano, se asignó un rol central al empresario innovador como impulsor competitivo, en el cual la búsqueda del lucro se constituía en el motor indirecto de la creación de conocimiento y la articulación entre creación de conocimiento como base de la "creación destructiva" y los mercados de trabajo (Shumpeter, 1983). Como sostiene (Baudelot y Leclercq, 2008), este carácter transformador no se restringe a productos o infraestructuras, sino que impacta en las competencias y capacidades de las personas que se tornan obsoletas en función de los cambios que se introducen. Esta nueva dinámica impacta en los procesos de formación del capital humano e introduce la renovación de conocimientos, la actualización curricular y sienta las bases de la educación permanente (Banco Mundial, 2003).

En esta línea en las últimas décadas, las concepciones primigenias sobre la innovación han recibido nuevos aportes que incorporan el rol del capital intelectual y la dinámica de transformaciones en curso de la dinámica societaria con la digitalización como eje de una "creación destructiva" que se apoya en la continua incorporación del conocimiento en los procesos de trabajo a través de dinámicas en red o en estaciones digitales (Tapscott, 2008, 2011). En esta línea se ha visualizado que los procesos de innovación tienen un creciente determinante derivado del uso de la microelectrónica y de las computadoras (David, 2003), como mecanismo para incorporar el valor agregado expresado en materia gris, en conocimiento e información. Como sostiene Tapscott (1993), el trabajo y también el capital pasan a ser el conocimiento aplicado a través de aplicaciones informáticas, trabajos en red y máquinas de control numérico.

Igualmente se desarrollan componentes no centrados exclusivamente en lo tecnológico, sino en multiplicidad de elementos societarios, entre ellos laborales, sociales, culturales, educativos, organizacionales y políticos, lo que eliminó el mero determinismo tecnológico y que revalorizan los determinantes de la infraestructura y de las estructuras socioculturales entre las cuales las educativas están presentes.

\section{LOS CAMBIOS EN LAS FORMAS DEL TRABAJO}

El mundo del trabajo está en transición hacia ambientes crecientemente digitales donde la informática es la herramienta del trabajo, las economías se internacionalizan, el comercio se apoya en red, la competencia se basa en la propiedad intelectual al fundamentarse en la incorporación de conocimientos (Aboites, 2008) y el capital humano es competencias y trabajo especializado (Reich, 1993). En el ámbito del ejercicio profesional, ello es más significativo, al producirse una transformación del trabajo que comienza a estar mediado por herramientas de tipo informáticas. Los especialistas universitarios, cuyo trabajo es diagnosticar y responder (diseñar, enseñar, prescribir, intervenir), en casi todos los campos laborales y disciplinarios, realizan sus actividades sobre la base de la información, por lo que el trabajo se asocia a las tecnologías como ámbito de gestión y de procesamiento informático.

Lo anterior facilita la incorporación de programas informáticos e internet en la educación, como mecanismo para adquirir esas competencias informáticas, informacionales y también las propiamente investigativas e innovadoras. Buscar y acceder a información pertinente, trabajar con periféricos informáticos más complejos, e instalar y configurar aplicaciones informáticas básicas y especializadas, se constituyen en requerimientos genéricos del mundo del trabajo para los profesionales y que imponen una educación orientada a facilitar la adquisición de estas competencias básicas y especializadas (Rama, 2010)

La incorporación de herramientas y aplicaciones informáticas en el proceso de enseñanza sigue múltiples líneas destacándose su aporte en el mejoramiento del proceso de enseñanza y su contribución a la apropiación de las dinámicas 
del trabajo de los distintos profesionales. Esta incorporación de las TIC en la dinámica de la enseñanza en todas sus dimensiones dentro y fuera del aula es vista como un cambio del paradigma educativo. En Europa, el Libro Blanco de la Universidad Digital 2010 (Laviña \& Mengual, 2008) visualiza como un cambio de paradigma la incorporación de estas tecnologías sobre la dinámica educativa por sus dimensiones diferenciadas. Ellas las define como superación y refiere entre ellas al abandono del calendario académico por uno todo el año; a la superación del aprendizaje terminal por un aprendizaje continuo; la sustitución del libro como medio principal de información frente a internet; la sustitución de la entrega en clase de los contenidos instruccionales por una que se realiza en todos lados; o la superación de los ladrillos por bytes como infraestructura educativa y espacio de comunicación. Sin embargo, esta nueva dinámica no se reduce a la educación a distancia como modalidad que usa tecnologías con intensidad, sino que crecientemente impacta en la educación presencial que se transforma a través de su digitalización.

\section{LAS BASES DE LA ACTUAL INNOVACIÓN EDUCATIVA}

La innovación educativa ha asumido una amplia variedad de formas dado su carácter sistémico. La mayoría de enfoques tienden a concordar en su carácter diverso como un fenómeno cultural, por lo que es difícil considerar una linealidad en su proceso, dada la circularidad y simultaneidad de los procesos (Ortega, 2007).

Siguiendo el enfoque de Shumpeter de la "creación destructiva" como centro de la innovación, en el sector de la educación, ello se produce a partir de la introducción de la tecnologías de información y comunicación, cuyas derivaciones impulsan múltiples transformaciones en las instituciones, en los accesos, en las formas de enseńanza o en los recursos de enseñanza ${ }^{4}$. Las tecnologías digitales facilitan la creación de nuevos productos, procesos y servicios, que tornan obsoletos los anteriores me-

4 Siguiendo el enfoque de Yip, (1995) de impulsores determinantes en las transformaciones de los procesos sociales, se ha verificado el impulso tecnológico como el más significativo en los cambios educativos en la educación a distancia (Rama, 2012). canismos de formar competencias y de apropiarse de los conocimientos. Al igual que en la sociedad, la innovación y el aumento de la productividad (medida por aprendizajes de competencias) se asocia a la expansión de la microelectrónica y la programación informática.

En el sector educativo la calidad no mejora sustancialmente agregando factores (más docentes, aulas o recursos), sino por la innovación de sus procesos de enseñanza y de las dinámicas del aprendizaje. Más allá de las innovaciones a nivel del aula o de métodos más idóneos, la innovación tiene un carácter sistémico y cultural, ella ha estado asociada también históricamente al desarrollo de los recursos de aprendizajes. Estos han ido evolucionando desde el uso del libro, del libro de textos, la guía didáctica, el video y que con la aparición de la digitalización y la creación de ambientes digitales han asumido nuevas expresiones al permitir una mayor interacción y eficiencia. Tales avances se muestran en recursos de aprendizaje con mayor interacción gracias a los software así como hipertextos, accesos en red, uso de multimedia, traducción automática de los contenidos, ambientes colaborativos y sin duda plataformas digitales.

En general las innovaciones educativas han sido continuas en la dinámica educativa. Podemos diferenciarlas en sus expresiones en innovaciones de de productos (carreras), formas (interdisciplinaridad), niveles (postgrados, etc.), pedagogías (aprendizaje basado en problemas (ABP), mapas conceptuales, estudios de casos), modalidades (educación a distancia y continua), ámbitos (campus universitarios, sedes o plataformas virtuales), currículos (estudios generales, pasantías preprofesionales, enfoques por competencias); estudiantes y docentes (extranjeros, a distancia), organización institucional (departamentos, unidades temáticas, sedes), etc. (Rama, 2009a). Igualmente podemos verificar procesos de innovación educativas tales como nuevos mercados y tipos de estudiantes a través de la educación transfronteriza, nuevas formas de organización y gestión educativas como franquicias, funcionamiento en redes o estructuras departamentalizadas, unidades de problemas o ciclos de estudios generales, incluso hasta nuevas fuentes de acceso a docentes o tutores de otros países. 
La innovación educativa expresada en pedagogías y didácticas, se asocia contemporáneamente a la incorporación de las TIC en la enseñanza, el aprendizaje y la gestión. Éstas son el factor más dinámico de la educación, impulsando una nueva dinámica de los aprendizajes y de las configuraciones organizacionales, y a la vez son la base de la irrupción de una pedagogía informática. Esta línea de innovación está marcada por cambios en los recursos de aprendizaje que tradicionalmente se ha referidos como productos y servicios de las industrias culturales y cuya intensificación de contenidos orientados al aprendizaje, así como la digitalización, las ha ido transformando en industrias educativas (Rama, 2003, 2004). Estas muestran una diversidad de manifestaciones tales como herramientas de trabajo docente y estudiantil, como los materiales de escritura, cuadernos, reglas, compases; recursos informacionales con contenidos como mapas, libros, diccionarios, enciclopedias, revistas, periódicos, videos, discos; y recursos de experimentación, tales como los aparatos que permiten la realización de actividades prácticas que refuercen los procesos de aprendizaje de conceptos, a través de instrumentos como microscopios o calculadoras.

En educación una de las innovaciones centrales están asociadas a estos diversos recursos de aprendizaje y al aumento de su intensidad de uso en el aprendizaje derivado de ellas, lo cual los consolida como productos de una industria educativa y por ende diseńados para crear aprendizajes y competencias. Con la digitalización, en tanto permite convergencia, mayor interacción y programación previa, se refuerza su articulación a la educación y se expanden como objetos de aprendizaje interactivos focalizados en el rol de apoyar el autoaprendizaje en tanto envases con contenidos. La digitalización permite la virtualización de los recursos de aprendizaje y la generación de nuevos, como por ejemplo el software e internet.

Las herramientas del aprendizaje son resultado de la "creación destructiva" que promueve la virtualización. Cambian los productos, las capacidades y los procesos de enseñanza, lo cual sienta las bases de la irrupción de una "pedagogía informática" como disciplina que organiza el proceso educativo en función de las posibilidades que brindan los hardware y software al aprendizaje

Ha habido una evolución continua de las innovaciones educativas. El libro como envase del conocimiento, históricamente fue una de ellas, y a la vez permitió la construcción de modelos educativos en el cual el aprendizaje se logra apoyado en las industrias culturales. Con ello se logra una apropiación con mayor profundidad y reflexividad ante el discurso oral. El seminario, base de la propuesta de la Universidad de Humboldt nació como una dinámica educativa basada en la discusión específica sobre un libro, mostrando un aprendizaje no basado sobre el trabajo vivo docente en el momento, sino sobre el producto, el libro en este caso, resultado de un trabajo intelectual previo. Este ha sido un componente de otros cambios, como el currículo, la tiza y el pizarrón, o de repertorios de recursos como las bibliotecas, y cuya evolución marcó el pasaje desde las industrias culturales a las industrias educativas.

Entre las innovaciones han sido un centro el desarrollo de los recursos de aprendizaje que tiene su hito con la irrupción del libro de texto y las guías didácticas, que significaron una transformación respecto al libro tradicional concebido de manera exclusiva como unidad de información. El libro de texto incluye se conforma como un recurso educativo de apoyo al autoaprendizaje y a la labor docente. No sólo es trabajo muerto, sino trabajo docente muerto el adicionamiento de componentes didácticos y pedagógicos. El pasaje del libro tradicional al libro de texto se dio mediante la inclusión del trabajo de especialistas en aprendizaje e implicó agregar síntesis de los contenidos, preguntas, extractos de otros contenidos, glosario de palabras, definición de conceptos, mapas conceptuales explicativos, referencias de terceros, mapas, esquema, así como preguntas y respuestas, ejercicios para el trabajo y diseños especiales de los contenidos. Como recurso de aprendizaje, contribuyó también a un cambio en el rol del docente que pasó a tener un papel más enfocado a transmitir marcos conceptuales, apoyar en la selección de esos recursos, dar respuestas y contextos a los autoaprendizajes basados en el libro y definir los objetivos y las formas de evaluación.

La innovación impactó en el mundo del trabajo 
docente y promovió un mayor cambio en la relación entre el trabajo vivo, en tanto acción directa como servicio educativo, hacia una expansión del trabajo muerto, en tanto trabajo previo de producción de bienes para producir el servicio educativo. La educación es una función de producción, inicialmente basada sólo en trabajo vivo y que ha ido cambiando al incorporar trabajo muerto (recursos de las industrias educativas) y cuya lógica está dada por la combinación más eficiente en términos de aprendizaje entre el trabajo vivo (docente) y el trabajo muerto (recursos de aprendizaje). Con lo digital, el trabajo docente directo comienza a ser sustituido y el aprendizaje se desarrolla crecientemente en forma exclusiva soportado en trabajo muerto (software y recursos abiertos de aprendizaje)

\section{LA VIRTUALIZACIÓN COMO LA INNOVACIÓN EDUCATIVA DOMINANTE}

Con creciente intensidad, una de las innovaciones más significativas en los últimos años, en lo que atañe a la dinámica educativa, es la digitalización y la virtualización de los procesos de enseñanza, en tanto permite nuevas combinaciones de componentes educativos, nuevos productos, nuevas interacciones y recursos de aprendizaje, junto con nuevos mecanismos de transmisión y construcción de conocimiento así como de evaluación de aprendizajes.

La estrategia para mejorar el aprendizaje en el contexto tecnológico contemporáneo implica potenciar el uso de internet, tener un enfoque por competencias, estimular la interacción múltiple desde plataformas y permitir apropiar los conocimientos mediante diversidad de recursos de aprendizaje. A ello se agregan hipervínculos, multimedia y aplicaciones informáticas. La incorporación en el uso de recursos informáticos como base de los aprendizajes para lograr adquirir competencias en forma más eficaz, implica aumentar el acceso tanto a la información, a recursos didácticos y a los diversos portadores de conocimientos. Tal objetivo se centra en una amplia como diferenciada interacción, y precisa la existencia de multiplicidad de recursos de aprendizaje específicos que potencien un mayor trabajo estudiantil y la construcción de redes de aprendizaje. Ello da sustentación a las pedagogías informáticas. García Aretio (2007) por ejemplo sostiene que hay seis enfoques en la educación virtual: el magistrocéntrico, el logocéntrico, el paidocéntrico, el tecnocéntrico, el interactivo y uno último que denomina integrador.

Se sostiene que sin intensificar y diversificar las interacciones, no se puede superar los modelos de enseńanza tradicionales e incorporar modelos centrados en los estudiantes y sus aprendizajes. La propia formación de competencias en el entorno virtual es aquella que alcanza los resultados a través de una interacción múltiple, con recursos de aprendizaje digitales, interfaces especiales con los sistemas informáticos, aplicaciones de práctica y autoaprendizaje que simule la realidad e interacción en red con otros estudiantes y profesores. Ello obliga una diversidad, una calidad y una lógica virtual de las interacciones.

La virtualización educativa impulsa además la transformación del aula y el aprendizaje presencial, a través de la introducción de plataformas de aprendizaje, herramientas y aplicaciones informáticas, interfaces hombre computadoras del tipo ratón (mouse) o láser, y del pizarrón que pasan a ser pantallas digitales con multiplicidad de posibilidades expresivas, entre las cuales está su conexión a internet, televisión, visualización de slides y de video. Con ello se pasa del aula al laboratorio como ámbito del aprendizaje.

La base de muchas de las interacciones es, sin duda, internet y las plataformas dentro o fuera de la nube, que impone un cambio en la metodología de enseñanza para impulsar un proceso de aprendizaje centrado en el estudiante con un enfoque por competencias y con diversidad de recursos en red fuera del aula. Aumentar la interacción mediante pedagogías informáticas busca superar el mero acceso a la información para focalizarse en la adquisición de competencias. El propio objetivo de buscar información se debe articular a diversidad de formas de interacción que mejoren, a la vez, la cartera de competencias. La incorporación de componentes informáticos facilita esa mayor interacción, una dinámica más articulada entre la teoría y la práctica y una mejor calidad de dichas 
interaccionas resultado de la cantidad y diversidad de los recursos de aprendizaje. En este contexto, el conectivismo se transforma en uno de los nuevos basamentos conceptuales de la pedagogía informática, que contribuye a superar el modelo unidireccional (profesor-estudiante) y lograr mejores aprendizajes con la inclusión de una interacción multidireccional. (Siemens, 2004)

\section{LA PEDAGOGÍA INFORMÁTICA}

La pedagogía informática, constituye una práctica para lograr el aprendizaje y también en una disciplina aplicada para organizar el proceso educativo tomando en cuenta las posibilidades que brinda la programación informática en el actual contexto social y tecnológico. No es el mero resultado de los recursos digitales sino de su articulación y de las formas educativas de su uso en la enseñanza y el aprendizaje. Es una actividad centrada en transmitir diversidad de información y conocimientos, promoviendo la adquisición de competencias con herramientas informáticas de comunicación e información con programación y promoviendo la creación de redes de colaboración con intensidad en el uso de redes de trabajo. Es una expresión de los cambios con las tecnologías de comunicación y la digitalización en lo educativo, construyendo lógicas de la enseñanza con mayor intensidad en la utilización de recursos didácticos y aplicaciones informáticas ajustadas a las particularidades de los estudiantes. Como pedagogías, se nutren de los aportes de varios campos de la ciencia y de otras disciplinas, pero sus métodos y bases se apoyan en el uso intensivo de las TIC, el cognitivismo, la neurociencia y el conectivismo. Al sostenerse que el aprendizaje puede residir en dispositivos no humanos y que el aprendizaje es conectar nodos distintos (Siemens), o que el aprendizaje significativo depende del material con el cual se aprende, y que este se produce cuando "se conecta" con un concepto relevante (imagen, símbolo, etc. (Ausubel, 1983)

La pedagogía informática tiene una alta capacidad de aprendizajes significativos por su capacidad de reproducir la realidad, de acceder a diversidad de situaciones y de integrar la diversidad de conexiones y de aportes de saberes. Utiliza las potenciali- dades de la programación e interfaces ajustados a los particularismos de contenidos, competencias y personas, y permite aprendizajes más eficaces para adquirir competencias (ej. personas con discapacidad, estudios de casos, ABP, simulación, telecirujías a través de robot, etc.).

La pedagogía informática parece darse con alta intensidad en asignaturas profesionalizantes, con la transformación de asignaturas (teóricas) a módulos articulados de teoría y práctica. En tal sentido se asocia a enfoques curriculares no basados en transferencia de conocimientos sino centrados en adquisición de competencias. Ella no implica una reforma completa a la vez, sino que se expande al interior del currículo mediante la introducción de asignaturas con mayores dinámicas de uso de aplicaciones informáticas que reproducen el ejercicio laboral, la formación de dinámicas de aprendizaje basadas en ellas, en nuevas capacidades docentes y en nuevas formas de evaluación con laboratorios de prácticas o de simulación.

El aula tradicional se transforma con la incorporación de hardware y software como base de las pedagogías informáticas. Estas promueven dinámicas de enseñanza presenciales en laboratorios y que son realizadas en entornos virtuales a través de computadores. En éstas la enseñanza y el autoaprendizaje se hacen juntos, propiciando una mejor formación de competencias a través de recursos didácticos interactivos en la red y con evaluaciones informatizadas. Este entorno educativo apoyado en plataformas y web, impone un cambio del currículo e impulsa la informatización administrativa, de inscripción, entrega de trabajos, conocimiento de notas y programas, asignación de aulas, laboratorios y docentes, seguimiento tutorial y acceso a recursos y bibliotecas digitales, como parte de un proceso en el cual el aula presencial se electroniza y se virtualiza.

La irrupción de las "pedagogías informáticas" es la expresión de la virtualización y digitalización, con una nueva epistemología del conocimiento (Ugas, 2005) que facilita una articulación con enfoques curriculares del tipo de "saber hacer". Su mayor intensidad se produce en las asignaturas y contenidos profesionalizantes, ya que en éstas es significativo el análisis y procesamiento de datos que 
reproducen el ejercicio laboral, con laboratorios de prácticas o de simulación, así como en asignaturas teóricas y aplicadas que crean conocimientos a través de la investigación y que, por ende, requieren procesar información con mayor eficacia mediante instrumentos digitales.

Esta pedagogía tiene limitaciones, pero sus resultados en el aprendizaje son muy superiores porque permiten ingresar a una educación haciendo. Obviamente, es éste un proceso que depende de los campos disciplinarios y de la complejidad de las herramientas informáticas, así como del propio desarrollo de estas tecnologías. En esta línea, Silva (2012) analiza el resultado de aprendizajes en la disciplina de "Estadística" entre un grupo de estudio que no tuvo pedagogías informáticas y otro cuyo aprendizaje se realizó utilizando herramientas informáticas. El estudio constata cómo las pedagogías informáticas permiten aprendizajes más significativos de ese grupo, medidos por las mismas pruebas. En su evolución, éstas superan los modelos de estímulo respuesta, el simple concepto de acceso a la información, o de interacción pasiva, para permitir evolucionar hacia herramientas de formación de competencias colaborativas con mayores niveles de interacción y representación de la realidad y del trabajo de ámbito laboral. Como se constata en el estudio de Silva, los distintos softwares de autoaprendizaje se convierten en herramientas que proporcionan niveles superiores de aprendizaje. Ello, sin duda, tiene, y tendrá, diversas fases. Para algunos autores (Ruiz-Velasco, 2006), incluso la robótica pedagógica se constituye en una forma superior de la propia pedagogía informática que facilita una mayor complejidad de la propia automatización pedagógica como herramienta de aprendizaje. Para estos autores, "uno de los principales objetivos de la robótica pedagógica, es la generación de entornos de aprendizajes heurísticos, basados fundamentalmente en la actividad de los estudiantes, los cuales podrán concebir, desarrollar y poner en práctica diferentes robots educativos que les permitirán resolver algunos problemas”. En tal sentido, plantean que "la robótica pedagógica privilegia el aprendizaje inductivo y por descubrimiento guiado". Los autores dan soporte novedoso a los enfoques constructivistas al sostener que la inducción y el descubrimiento guiado y programa- do permitirán a la vez a los estudiantes construir su propio conocimiento. Para otros por su parte Odorico (2004), la robótica pedagógica se asocia a la lógica del aprendizaje de la simulación más allá de ser un hardware o un software.

La pedagogía informática se expande ante las dificultades de reproducir en la práctica realidades cada vez más diversas; la dificultad de supervisar educativamente las pasantías y las prácticas estudiantiles; y el aumento y diferenciación del conocimiento, así como de la programación y de interfaces ajustadas a los particularismos de contenidos, competencias y personas como por ejemplo las personas con capacidades especiales (Rama, 2013). Con ella, los sistemas educativos pasan de la valorización de la práctica presencial para alcanzar el aprendizaje, al uso de la informática como realidad y simplificación virtual para adquirir las competencias.

Varias son las causas de la virtualización educativa que está llevando a la transformación de las tradicionales aulas y su enseñanza tradicional presencial de tiza, lengua, pizarrón y bibliotecas tradicionales así como de las instituciones que eran expresión de sistema de enseñanza, repetitivo, memorístico y catedrático. Igualmente acontece con aquellas pedagogías tradicionales. El aumento de los conocimientos y de la competencia entre las instituciones educativas y en los diversos mercados laborales favorece la realización de reformas educativas que propicien innovaciones educativas para transitar desde el enfoque en el "saber" a los múltiples "saber hacer" a través de la virtualización, planteando un modelo educativo centrado en el aprendizaje con aumento de prácticas profesionales mediante herramientas informáticas en ambientes virtuales, en tanto el propio trabajo profesional de diagnóstico y respuesta se hace a partir de la información obtenida con nuevos hardware y software.

\section{EL APRENDIZAJE REAL}

El enfoque de las competencias profesionales, en su clasificación más simple, se separa en competencias epistemológicas de tipo teóricas y competencias aplicadas o prácticas, pero en un contexto donde lo significativo es su unidad, más allá de 
los múltiples desagregamientos que propenden a identificar las características y habilidades que mejor se ajustan a mercados profesionales cada vez más complejos en términos de información para la toma de decisiones. Ello implica poner en el centro el laboratorio digital como aula educativa que integra lo presencial y la computadora con una creciente revalorización de la práctica.

Históricamente, la adquisición de competencias y una de las primeras innovaciones educativas fueron las prácticas, que incorporaron como ambientes de aprendizajes a los ambientes reales. Ello se ha expresado en tiempo más presente en la incorporación de las prácticas profesionales, las pasantías o los servicios sociales. Éstas han sido, en general, muy complejas de gestionar, supervisar, instrumentar y evaluar, con diversidad de modalidades de gestión, de complejidades del derecho intelectual, con nuevas formas de evaluación comparativas, externas y automatizadas, y altos costos, que han dificultado su instrumentación en la mayor parte de los centros universitarios. Las metodologías de estudios de casos (EdC) o el aprendizaje basado en problemas (ABP) se han convertido en dinámicas pedagógicas que plantean un nivel práctico más cerca a los entornos reales y que de hecho implican una superación de la dinámica tradicional catedrática en el aula. Sin embargo en tanto el método de estudio de casos se enfoca a analizar apenas una situación poco generalizable, al tiempo que en el aprendizaje basado en problemas, se parte de interrogantes que obedecen también a un hecho específico, a partir de donde se plantean posibles soluciones ${ }^{5}$. Estos métodos contienen baja generalización y por ende su valor en el aprendizaje es relativo. Sin embargo, con la programación informática, pasan a permitir incorporar cambios y las variables de los casos y problemas, con lo cual aumentan sus eficacias educativas.

El ABP reafirma el pensamiento complejo, mejora los vínculos entre aprendizajes teóricos y prácticos, incrementa las oportunidades de colaboración e interacción e intensifica su mayor potencia pedagógica con el uso de nuevas tecnologías como las propias simulaciones por ordenador. (Araujo

5 http://portal.educar.org/foros/estudio-de-casos-y-aprendizaje-basado-en-problemas
\& Sastre, 2008). En contraste con la enseñanza tradicional, que se basa en exposiciones catedráticas, el EdC y el ABP se dan en grupos que trabajan juntos en el estudio de un problema, y se enfocan a generar soluciones viables. Así, asumen una mayor responsabilidad sobre su aprendizaje, perspectivas multidisciplinarias y nudos problemáticos como ejes del aprendizaje. Son parte de una dinámica de la enseñanza pertinente que pasa desde las disciplinas a los departamentos, y de éstos a los nudos problemáticos como ejes del aprendizaje (Buarque, 2004)

El ABP acerca la educación al trabajo y tiende a utilizar el mundo del trabajo y los espacios de problemas como lugares de aprendizaje, al facilitar una dinámica de enseñanza y aprendizaje que supera los meros contenidos teóricos en tanto están perdiendo relevancia y significado ante la renovación de los conocimientos. Esta modalidad busca que, a la par de la adquisición de conocimientos, se construyan las capacidades y hábitos acordes con esos saberes.

Desde estos enfoques educativos, los estudiantes abordan problemas reales o hipotéticos en grupos pequeños y con la supervisión de un tutor. El estudio de casos focaliza el aprendizaje en la interacción y trabaja con problemas concretos y su enseñanza se centra en el estudiante (Brezzo, 1971). El material didáctico en el estudio de casos se compone de descripción de situaciones reales en las que el caso sustituye al mero ejercicio y presenta una situación más compleja y cercana a la realidad al agregar más elementos para el análisis. Tal lógica de "casos" se generaliza y mejora con el uso de las pedagogías informáticas, al aumentar su complejidad y volúmenes de variables y de información, gracias a lo cual dejan de ser casos específicos para concebirse como estudios de escenarios con diversidad de situaciones y problemas, y por ende incorporado y modificando variables en la representación de lo real y, por ende, acercarse más a la diversidad de la realidad que por definición es sistémica y compleja (Morin, 1988, 2000, 2002)

Con la programación informática se amplían las potencialidades de los métodos y criterios que sustentan la EdC y el ABP, al facilitar una nue- 
va dinámica a través de sistemas de simulación y creación de realidades virtuales. Con ellas se forman competencias mediante múltiples aplicaciones digitales que simulan la realidad y los ambientes y situaciones de trabajo, y al mismo tiempo, permitir construir múltiples escenarios hipotéticos, superando los aprendizajes teóricos y realizar aprendizajes constructivistas e interaccionistas en un contexto conectivista. La programación permite así complejizar el análisis de casos e impulsar un aprendizaje interactivo con variados escenarios posibles, incluyendo las incidencias de los cambios en las variables de una situación específica.

\section{CONCLUSIONES: DEL CAMBIO TECNOLÓGICO AL CAMBIO PEDAGÓGICO}

La transformación de la lógica presencial y la incorporación de tecnologías ha sido un proceso continuo que ha ido transformando la enseñanza catedrática. Desde la propia aula y el libro, la biblioteca y el currículo, o el estudio de casos o la enseñanza basada en problemas, se han impuesto cambios en el modelo de enseñanza aprendizaje tradicional. Con los ambientes virtuales los cambios asumen una dimensión educativa muy superior y plantean cambios que visualizan una nueva pedagogía informática. Esta no es un mero resultado mecánico de la incorporación de las TIC, las cuales son condición necesaria pero no suficiente. La incorporación de tecnologías en las aulas no tiene necesariamente por si solas efectos positivos en el rendimiento de los aprendizajes ni mucho menos en la modificación de las pedagogías de enseñanza, pero su propia incorporación comienzan a mostrar ineficiencias de las prácticas anteriores, y torna obsoleta muchos de los procesos de enseñanza.

La irrupción de la pedagogía informática es una derivación de las posibilidades que brinda la digitalización fundamentalmente de los recursos de aprendizaje, del acceso en red y de las aplicaciones informáticas. La pedagogía se orienta a superar la dinámica unidireccional, catedrática y centrada en los docentes, promoviendo a la vez la remodelación del aula que pasa a ser digital. Se expresa en plataformas y ambientes de aprendizaje para apo- yar y gestionar el proceso de enseńanza, bibliotecas con acceso en red dentro del aula, asesoría tutorial en la propia aula, pizarrones electrónicos, presentaciones virtuales, mapas conceptuales virtuales, etc., así como la inclusión de otros docentes a través de la red y videoconferencias. Algunas veces el propio trabajo docente se realiza desde fuera del aula, con pizarrones digitales conectados a redes, $y$ los alumnos vinculados en redes de computadores con el docente. Se desarrolla una dinámica de autoaprendizaje mediada por aplicaciones informáticas, se verifica la originalidad o plagio mediante aplicaciones informáticas e inclusive se evalúan los aprendizajes a través de sistemas digitales de selección múltiple choice o trabajos colaborativos en Web 2.0 o wikis. Irrumpen además múltiples interfaces digitales (joystik, ratón, láser, etc.).

La computadora (Silvio, 2000) con sus diversas interfaces y aplicaciones específicas a los distintos cambios de competencias, se convierte en el instrumento del proceso educativo y fija los parámetros de estas pedagogías informáticas que han ido evolucionando y cambiando la dinámica de las clases presenciales y a distancia. El incremento de la cantidad de computadores por estudiante mide el proceso en curso y expresa una mayor intensidad de las pedagogías informáticas, entre otras, a través de conectividad para el acceso, interacción estudiantes-recursos instruccionales o estudiantes-profesores, de autoaprendizaje mediante aplicaciones específicas y que transforma los modos de transferir y apropiarse de información y construir las competencias.

Se produce una convergencia entre la lógica presencial y la virtual, en la cual las plataformas se constituyen en ejes centrales del proceso educativo tanto para estudiantes como para docentes (Legizamón \& Sánchez, s/f). Facilitan el aprendizaje apoyado en la Web y en herramientas, y propician el cambio del modelo lineal, catedrático, memorístico y unilateral al aumentar el tránsito desde materiales lineales, como los libros, a lógicas en red de hipertextos; desde dinámicas educativas con interacción baja unidireccional a un aumento de su intensidad incorporando múltiples niveles de interacción; desde microbibliotecas a internet; desde aprendizaje pasivos a autoaprendizajes sobre aplicaciones informáticas. Inclusive desde el libro 
a las revistas académicas en red y de acceso abierto bajo Open Journal System (OJS). Un proceso de "creación destructiva" que va sacando del centro del aprendizaje la tiza, la lengua y el pizarrón y a la vez cambia el rol docente al sustituirse partes crecientes del trabajo vivo en la enseñanza y que a la vez aumenta el uso del trabajo muerto docente a través de múltiples recursos de aprendizaje realizados previamente con trabajo integrados en equipos más complejos y profesionales con diversidad de tareas y diferenciadas competencias y centrados más en el autoaprendizaje. Estas innovaciones son espacios de "creación destructiva" impulsados por hardware y software que transforman el aula y su contexto; con internet que modifica la forma de acceder a la información y se constituye en nueva red de recursos; con docentes que hacen su trabajo cada vez menos frente a los alumnos y más frente a las computadoras y desde trabajos solitarios a dinámicas en equipos con productos previos al trabajo en el aula. Ello es la base de la conformación de una nueva pedagogía informática que a la vez se sostiene en nuevos paradigmas del aprendizaje.

\section{REFERENCIAS BIBLIOGRÁFICAS}

Ausubel, D. (1983). Teoría del aprendizaje significativo. Recuperado de http://www.educainformatica.com.ar/docentes/tuarticulo/educacion/ausubel/index.html (Acceso: 28/06/2014)

Aboites, J. y Soria, M. (2008). Economía del conocimiento y propiedad intelectual. Lecciones para la economía mexicana. UAM, Siglo XXI, México

Albornoz, M. (2009). "Indicadores de innovación: las dificultades de un concepto en evolución”. Revista CTS (Revista Iberoamericana de Ciencia, Tecnología y Sociedad (CTS), 13, (5) 9-25.

Araujo, U. y Sastre, G. (coordinadores) (2008). El aprendizaje basado en problemas. Una nueva perspectiva de la enseñanza en la universidad. Barcelona, Gedisa Editorial.

Banco Mundial (2003). Aprendizaje permanente en la economía global del conocimiento. Washington: Alfaomega, Banco Mundial.

Baudelot, C. y Leclerq, F. (Dirs). (2008). Los efectos de la educación. Buenos Aires, Del Estante.

Brezzo, R. (1971). El método de casos en la enseñanza de la administración. Montevideo, Universidad de la República, Facultad de Ciencias Económicas y de Administración, Cuaderno 61.

Bates, T. (2001). Como gestionar el cambio tecnológico. Gedisa, Barcelona.
Buarque, C. (2004). The university at a crossroad. World Conference on Higher Education +5 at UNESCO Paris, UNESCO, Ministerio de Educación. Recuperado de http:// unesdoc.unesco.org/images/0013/001363/136394eo.pdf

Busch, V. (1999), Ciencia, la frontera sin fin. Informe al presidente, julio de 1945, Redes No 14, (7), Buenos Aires, Universidad Nacional de Quilmes.

Carnoy, M. (2006). Economía de la educación. Editorial UOC, Barcelona.

Coriat, B. (1992). El taller y el robot. Ensayos sobre el fordismo y la producción en masa en la era de la electrónica. Siglo XXI, México.

Daniel, J. (2003). Educação e tecnologia num mundo globalizado. UNESCO, Brasília.

David, P. (2003). La computadora y la dinamo. La paradoja de la productividad moderna en un espejo no muy lejano", en Chesnais, Francois y Neffa, J. C. (compiladores), Ciencia, tecnología y crecimiento económico, Buenos Aires, CEIL-PIETTE CONICET.

Easterly, W. (2002). O espetáculo do crecimiento, Rio, Ediouro.

García, A. (coordinador), Corbella, \& Domínguez (2007). De la educación a distancia a la educación virtual, Barcelona, Ariel

Laviña, J. y Mengual, L. (2008). Libro Blanco de la Universidad Digital 2010.Ariel, Madrid. Recuperado de

http://bases.cortesaragon.es/bases/NDocumen.nsf/ b4e47719711a1d49c12576cd002660cc/6cbb83eb3e6fbf54c1257576002ffb50/\$FILE/eLibro\%20Blanco \%20 de\%20la\%20Universidad\%20Digital\%202010_cap1.pdf

Legizamón, M. Á. y Sánchez, O. (s/f). Creación de ambientes virtuales como apoyo en el desarrollo de asignaturas al interior de la licenciatura en Informática Educativa. Programa académico de la Universidad Pedagógica y Tecnológica de Colombia, Tunja. Recuperado de https:/www.yumpu.com/ es/document/view/14250244/creacion-de-ambientes-virtuales-colombia-aprende

Montoya, O. (2004). Schumpeter, innovación y determinismo tecnológico, Scientia et Técnica, año X, núm. 25, agosto. Universidad Tecnológica de Pereira, Colombia.

Morin, E. (1988). El método. el conocimiento del conocimiento, Cátedra, Madrid.

Morin. E. (2000). Los sietes saberes necesarios a la educación del futuro. IESALC, Caracas.

Morin, E. (2002). La cabeza bien puesta. Repensar la reforma, reformar el pensamiento. Nueva Visión, Buenos Aires

Odorico, A. (2004). Marco teórico para una robótica pedagógica, Revista Informática Educativa y Medios Audiovisuales, 1, (3), 34-46, Universidad de Buenos Aires.

Ortega C, Pedro et al. (2007) Modelo de innovación educativa. Un marco para la formulación y el desarrollo de una cultura de la innovación, Revista Iberoamericana de Educación a Distancia, 10, (1) 145-173. 
Pérez, C. (1996). Cambio técnico, reestructuración competitiva y reforma institucional en los países en desarrollo, Santiago de Chile, CEPAL/CLADES.

Pérez, C. (2004). Revoluciones tecnológicas y capital financiero, México, Siglo XXI

Rama, C. (2003). Las industrias culturales en la globalización digital. EUDEBA, Buenos Aires.

Rama, C. (2004). El nuevo paradigma de la educación y el papel de las industrias culturales, en Marta Mena (compiladora), La educación a distancia en América Latina, modelos, tecnologías y realidades, Puebla, México, El Colegio de Pueblo, ICDE, CREPEPP, 285-293.

Rama, C. (2007. La despresencialización de la educación superior en América Latina: ¿tema de calidad, de cobertura, de internacionalización o de financiamiento?, Revisa Apertura, 7, (6) 32 - 49. Recuperado de http://www.redalyc. org/articulo.oa? $\mathrm{id}=68800604$

Rama, C. (2009). Las tendencias del currículo, la tecnología y la gestión en el nuevo paradigma de la educación, Revista Mexicana del Bachillerato a Distancia. 1, (2), México, DF.

Rama, C2010). Paradigmas emergentes, competencias profesionales y nuevos modelos universitarios en América Latina, Puebla, Benemérita Universidad Autónoma de Puebla (BUAP).

Rama, Claudio (2010b). "La tendencia a la despresencialización de la educación superior en América Latina", Revista Iberoamericana de Educación a Distancia. 13, (1), 39 - 72. Recuperado de http://ried.utpl.edu.ec/images/pdfs/ vol13N-1/ried_13-1(definitivo).pdf

Rama, C. (2012). La reforma de la virtualización de la universidad. El Nacimiento de la educación digital. UNICARIBE, Santo Domingo.

Rama, C., (2013). "La educación virtual como la modalidad educativa para las personas con necesidades especiales". Revista Diálogo Educativo. Pontifícia Universidade Católica de Paraná (PUCP). Curitiba. Brasil. 13 (38), 325-345. Recuperado de http://www.redalyc.org/articulo. oa?id=189126039016

Reich, R. (1993). El trabajo de las naciones. Hacia el capitalismo del siglo XXI. Vergara, Buenos Aires

Rosemberg, N. (2003). Ciencia innovación y crecimiento económico, en Chesnais, Francois y Neffa, J. C. (compiladores), Ciencia, tecnología y crecimiento económico, Buenos Aires, CEIL-PIETTE CONICET

Ruiz-Velasco, E., Sánchez M. B., Freyre, A. Rodríguez, P., Martínez, J. V., García, L. Rosas, Y. Koyama, M., Velázquez, M. (2006). Robótica pedagógica: desarrollo de entornos de aprendizaje con tecnología, Universidad Nacional Autónoma de México (UNAM). Recuperado de http:// www.virtualeduca.org

Shumpeter, J. (1983). Capitalismo, socialismo y democracia. Aguilar, M. y Siemens, G. (2004). Conectivismo: Una teoría de aprendizaje para la era digital. Recuperado de http://es.scribd.com/doc/201419/Conectivismo-una-teo- ria-del-aprendizaje-para-la-era-digital.

Silva P., Edel G. (2011). Tecnologías en el aprendizaje de la estadística. Tesis doctoral. Inédita. Universidad de la Empresa, Montevideo.

Silvio, J. (2000). La virtualización de la universidad, ¿cómo podemos transformar la educación superior con la tecnología?, IESALC, Caracas

Skinner, B.J. (1979). Tecnología de la enseñanza. Labor, Barcelona

Tapscott, D. y Williams, A. D. (2011). Macrowikinomics. Nuevas fórmulas para impulsar la economía mundial. Madrid, Planeta.

Tapscott, D. y Williams, A. D. (2008). Wikinomics. La nueva economía de las multitudes inteligentes. Madrid, Paidós.

Tapscott, D. y Caston, A. (1993). Paradigm shift: the new promise of information technology. Universidad de Minnesota. Nueva York, McGraw-Hill.

Tedesco, J. C. (2008). Las TIC en la agenda de la política educativa. En varios, Las TIC: del aula a la agenda política. Buenos Aires, UNESCO-IIEP, UNICEF.

Tiffin, J. y Rajasingham, L. (2007). A universidades virtual e global. Porto Alegre, Artmed.

Ugas, A. (2005). Epistemología de la educación y la pedagogía. Caracas, IESALC.

Villa, L. (2001). Economía de la educación. Bogotá, Universidad de Los Andes.

Yip, G. (1995). Globalización: Estrategias para obtener una ventaja competitiva internacional. Bogotá, Grupo Editorial Norma.

Youssef A. Youssef y Ramirez, A. (2011). Social software in higher education: pedagogical models and universities strategies. Brasil: UNISUL 